\title{
Synthetic blends of volatile, phytopathogen-induced odorants can be used to manipulate vector behavior
}

\author{
Alexander A. Aksenov' ${ }^{1}$, Xavier Martini ${ }^{2}$, Weixiang Zhao ${ }^{1}$, Lukasz L. Stelinski ${ }^{2}$ and Cristina E. Davis ${ }^{1 *}$ \\ Mechanical and Aerospace Engineering, University of California, Davis, Davis, CA, USA \\ ${ }^{2}$ Department of Entomology and Nematology, Citrus Research and Educational Center, University of Florida, Lake Alfred, FL, USA
}

\section{Edited by:}

Jeffrey A. Riffell, University of

Washington, USA

Reviewed by:

Hong Lei, University of Arizona, USA

Jeremy J. Heath, North Carolina

State University, USA

\section{${ }^{*}$ Correspondence:}

Cristina E. Davis, Mechanical and Aerospace Engineering, University of California, Davis, One Shields

Avenue, Davis, CA 95616, USA e-mail: cedavis@ucdavis.edu
Volatile organic compounds (VOCs) are emitted from all plants and these VOCs are important means of communication between plants and insects. It has been documented that pathogen infections alter VOC profiles rendering infected plants more attractive to specific vectors transmitting these pathogens than uninfected plants, thus potentially aiding in pathogen propagation. Mimicking these chemical cues might enable insect attraction away from the plant or disruption of host finding behavior of the vector. However, the practical implications have not been fully explored. We used citrus, Diaphorina citri and huanglongbing (HLB) as a model host-vector-disease system because HLB threatens citrus production worldwide and is similar to other critical diseases of food crops, such as Zebra Chip affecting potato. We formulated a synthetic chemical blend using selected HLB-specific biomarker compounds, and tested the blend with the Attenu assay system for chemosensory proteins. The Attenu assay system is a procedure that identifies interactions between insect chemosensory proteins and their ligands. We found that mixtures of compounds mimicking the volatile profile of HLB-infected citrus can be bound by chemosensory proteins. Further investigation of these blends in laboratory behavioral assays resulted in development of a synthetic lure that was more attractive to $D$. citri than natural citrus tree volatiles. This strategy could provide a new route to produce chemical lures for vector population control for a variety of plant and/or animal systems and it may result in the development of a practical lure for monitoring vectors of disease, such as D. citri.

\section{Keywords: citrus greening, Asian citrus psyllid, lure, chemoattractant, VOC, odorants protein binding assays}

\section{INTRODUCTION}

Plants are known to communicate with one another and with insects by emitting bouquets of chemicals called volatile organic compounds (VOCs). These chemical cues are released, in some cases, in response to damage by insects (Kost and Heil, 2006). Plant-insect "conversations" have been investigated for approximately two decades and a great deal of progress has been made in understanding the mechanisms of their release. It is now clear that different plant species emit their own unique chemical blends and some chemicals have ubiquitous importance. Examples include methyl jasmonate and methyl salicylate (Rodriguez-Saona et al., 2011; Pierik et al., 2014), which are used in plant defense (among other roles). An important implication of understanding plant chemical signaling is the possibility of producing VOC blends that may manipulate or interfere with interactions between plants and insects for biological control and pest management, for example, via genetic engineering (Kos et al., 2013) or more traditional approaches of semiochemical application with controlled release devices (Witzgall et al., 2010b).

It has been documented that upon infection, pathogens can alter plant VOC output to attract vectors to the host, thus potentially aiding in pathogen propagation (Eigenbrode et al., 2002; McLeod et al., 2005; Mauck et al., 2010; Davis et al., 2012;
Shapiro et al., 2012). "Deceptive" attraction of vectors to infected plants from which they subsequently disperse may be conducive to enhanced pathogen transmission and may be honed through natural selection of vector behavior. For example, simultaneous manipulation of both plant odorant release and nutritional quality may result in initial attraction of herbivores to nutritionally sub-optimal, pathogen-infected plants followed by subsequent dispersal to and settling on nutritionally superior, non-infected counterparts (Mauck et al., 2010; Mann et al., 2012). This mechanism may drive pathogen spread throughout a community of plant hosts and has been termed the "deceptive host phenotype hypothesis" (Mauck et al., 2010). Here, we investigated whether it is possible to exploit this phenomenon for practical application to attract insect vectors by identifying and mimicking the chemical cues produced by pathogen-infected trees.

We have specifically targeted the Asian citrus psyllid, Diaphorina citri Kuwayama, which is the insect vector of Liberibacter species pathogens among citrus hosts. D. citri is a globally invasive species and, more importantly, a vector for Liberibacter species, including Candidatus Liberibacter asiaticus (CLas) (Grafton-Cardwell et al., 2013). CLas is one of the bacterial pathogens causing huanglongbing (HLB). This disease is considered the greatest threat to citriculture worldwide (Callaway, 2008) 
and is propagating through South and North America. Currently, management of HLB is mainly based on chemical control of D. citri populations (Grafton-Cardwell et al., 2013). However, overuse of insecticides may negatively affect the environment and is known to cause insecticide resistance in populations of $D$. citri (Grafton-Cardwell et al., 2013). The behavior of D. citri appears congruent with the "deceptive host phenotype hypothesis" given that D. citri are more attracted to CLas-infected plants than uninfected plants. Moreover, after initially settling on CLas-infected plants, $D$. citri subsequently disperse to nearby uninfected plants in search of a more nutritious host (Mann et al., 2012). Current trapping methods for D. citri rely on the use of yellow sticky traps without an associated olfactory lure. Unbaited traps capture D. citri from short distances and are marginally effective as monitoring tools (Grafton-Cardwell et al., 2013). Development of an attractant for D. citri would not only improve monitoring, but may also allow for development of other biorational tools, such as attract-and-kill or host finding disruption formulations.

\section{MATERIALS AND METHODS GC-MS AND qPCR ANALYSES}

Volatiles were collected from Hamlin sweet orange (Citrus sinensis L. Osbeck) trees using polydimethylsiloxane (PDMS)-based Twister $^{\mathrm{TM}}$ (GERSTEL, Inc.) sorbent beads as described by Aksenov et al. (2014). Trees were sampled at the University of Florida Citrus Research and Education Center (CREC), Lake Alfred, FL, USA. Infected and uninfected trees were selected by professional scouts and the infection status of the trees was confirmed using quantitative PCR (qPCR). The qPCR analysis for DNA extracts was conducted according to standard methods for detection of the CLas bacterium (Pelz-Stelinski et al., 2010). Four primers and one Taqman probe empirically designed based on $16 \mathrm{~S}$ sequences of CLas species were used (Pelz-Stelinski et al., 2010). The reverse primer (HLBr) used is specific to the genus Liberibacter and recognizes all three species within the genus.

Three independent studies were conducted to account for weather and seasonal variations in VOC production: winter (December, 2010), spring (March, 2011) and fall (September, 2011). The detailed description of the experimental protocol is provided in Aksenov et al. (2014). The volatile compounds captured by the Twister ${ }^{\mathrm{TM}}$ sorbent were thermally desorbed and analyzed by gas chromatography-mass spectrometry (GC-MS) as described in Skogerson et al. (2011). Briefly, we used a 6890 GC (Agilent Technologies, Santa Clara, CA) equipped with a thermal desorption unit (TDU, GERSTEL, Inc., Muehlheim, Germany) with a cryo-cooled injection system inlet (CIS4, GERSTEL, Inc.), and interfaced to the Pegasus IV time-of-flight mass spectrometer (LECO, St. Joseph, MI). The volatiles trapped using Twisters were thermally desorbed in the TDU in splitless mode. The desorbed analytes were cryofocused in the CIS4 inlet with liquid nitrogen $\left(-120^{\circ} \mathrm{C}\right)$, heated from -120 to $260^{\circ} \mathrm{C}$ and were analyzed on a Rtx-5SilMS column with a $10 \mathrm{~m}$ integrated guard column [95\% dimethyl/5\% diphenyl polysiloxane film; $30 \mathrm{~m} \times 0.25 \mathrm{~mm}$ (inside diameter) $\times 0.25 \mu \mathrm{m} \mathrm{d}_{\mathrm{f}}($ Restek, Bellefonte, PA)]. The GC oven temperature program was set as follows: initial temperature of $45^{\circ} \mathrm{C}$ with a $2 \mathrm{~min}$ hold, followed by a $20^{\circ} \mathrm{C} / \mathrm{min}$ ramp up to $300^{\circ} \mathrm{C}$ with a $2 \mathrm{~min}$ hold, and thereafter a $20^{\circ} \mathrm{C} / \mathrm{min}$ ramp up to $330^{\circ} \mathrm{C}$ with a $0.5 \mathrm{~min}$ hold with a constant $1 \mathrm{~mL} / \mathrm{min}$ flow of the carrier gas $(99.9 \% \mathrm{He})$. Mass spectra were acquired at 25 spectra/sec with a mass range of $35-500 \mathrm{~m} / \mathrm{z}$, with the detector voltage set at $1800 \mathrm{~V}$ and the ionization energy at $70 \mathrm{eV}$. Raw GC-MS data were pre-processed by Leco ChromaTOF software. The compounds were identified based on similarity of mass spectra and retention indices to that of the corresponding chemical standards (Skogerson et al., 2011). The chemical standards of the selected compounds were then purchased from Sigma-Aldrich (St. Louis, MO USA) and TCI America (Portland, OR USA) for development of a synthetic blend. If a compound did not meet the similarity score threshold (Skogerson et al., 2011), it was presumed unidentified and assigned a database entry number. The list of compounds, both identified and unidentified and their corresponding abundances, was generated for each sample. In the generated tables of compounds, every peak was normalized against the sum of the peak intensities.

\section{SELECTION OF CHEMOATTRACTANT COMPOUNDS}

The comprehensive lists of compounds produced by uninfected and infected sweet orange trees reported by Aksenov et al. (2014) were analyzed to select subsets of compounds for the use as chemoattractants in this investigation. In order to reduce the large number of compounds that differed between uninfected and infected trees to a single subset that was universal across varying stages of infection, infection subgroups with various severities of symptoms were combined into one "HLB" group. The student's $t$-test was then performed for each individual study with the alpha value set to 0.1 .

To further constrain the list of compounds from seasonspecific HLB biomarkers to those that can potentially discriminate uninfected and infected plants across different growing seasons, the list of biomarkers was narrowed down to only those that were found to discriminate the infection status during more than one season. The list of these biomarkers is given in Table S1 in the Supplementary Material [the unidentified compounds are given as Fiehn database entries available at (http://fiehnlab. ucdavis.edu/db/)].

\section{SCREENING D. CITRI CHEMOSENSORY PROTEINS WITH COMPOUND MIXTURES USING THE ATTENU ASSAY SYSTEM}

Insects use their chemosensory systems to interact with each other and their environment. The cells comprising the chemosensory apparatus can be accessed by odor molecules through the pores on the chemosensory bristle and hemolymph. The hemolymph, an aqueous solution, contains soluble chemosensory proteins such as odorant binding proteins (OBPs), sensory appendage proteins (SAPs) and odor degrading enzymes (ODEs) that are expressed by the insect. The OBPs bind hydrophobic odorant molecules thus promoting their aqueous solubility, while SAPs, shorter proteins, are involved in odorant transport. The odor molecule can interact with the proteins forming protein-ligand molecule complex. The complex can then bind chemosensory receptors, resulting in further molecular events that elicit recognition of the odor by the insect (chemosensory event). Interaction of the chemosensory proteins with odorant molecules can be studied in solution if the former are known and available. We 
investigated one odorant binding protein (DcOBP1) and four sensory appendage proteins (DcSAP1, DcSAP2, DcSAP3, and DcSAP4) from $D$. citri for which there is sequencing information for encoding OBPs or SAPs of $D$. citri (available at National Center for Biotechnology Information database). These chemosensory proteins have been identified, expressed and characterized by Inscent, Inc. The selected proteins represent classes of insect chemosensory proteins that are suitable as targets. For example, a direct correlation has been shown between OBP function and insect responses to odors in the malaria mosquito, Anopheles gambiae (Biessmann et al., 2010). Knockdown of OBPs has demonstrated detection of oviposition attractants in Culex (Pelletier et al., 2010). Also, it has been shown that two Drosophila species rely on a specific OBP to dictate important behaviors and that behavior can be attributed directly to the function of an OBP (Matsuo et al., 2007). For example, the Drosophila OBP, LUSH, is essential for mediating chemosensory responses and odor discrimination (Zhou et al., 2004) and the importance of these proteins in insect olfaction is well known (Swarup et al., 2011; Deng et al., 2013).

Protein binding of odorants was investigated using the Attenu assay, a proprietary high-throughput assay system developed at Inscent, Inc. that builds upon proven fluorescence-based techniques. Attenu is a fluorescence-based competition assay that relies on detectable fluorescence quenching to identify interactions between insect chemosensory proteins and their ligands (Pelosi et al., 2006; Biessmann et al., 2010). When a ligand displaces a fluor (fluorescent dye) from the binding pocket of an insect chemosensory protein, the resulting reduction in fluorescence signifies a binding event between the protein and that ligand. Thus, the assay allows high-throughput identification of whether a chemical of interest can be potentially detected at the peripheral nervous system level of an insect. The assay was used to screen the binding efficacy of the selected compounds. Typical assay conditions utilize $\sim 2-5 \mu \mathrm{M}$ of binding protein and $2-10 \mu \mathrm{M}$ of selected chemical compounds.

The goal of the screen was to determine which, if any, of the specific chemicals or mixtures identified could bind to either of the five chemosensory proteins from $D$. citri available at Inscent, Inc.: DcOBP1, DcSAP1, DcSAP2, DcSAP3, and DcSAP4. Each protein was screened at $4 \mu \mathrm{M}$ (DcOBP1) or $10 \mu \mathrm{M}$ (DcSAP1, DcSAP2, DcSAP3, and DcSAP4) correspondingly with every compound available (Table 1), as well as, an equimolar mixture of compounds, wherein each compound was present at $10 \mu \mathrm{M}$. Further screening of the compound mixtures that mimic biogenic abundances was conducted with the total concentration of all components combined at $10 \mu \mathrm{M}$, with molar ratios of each component corresponding to the values given in Table 1. The chemosensory proteins were assayed within DMSO as the solvent. Screening for binding was performed in triplicate and with appropriate controls in order to confirm possible significant interactions. The amount of tricosane greatly exceeds that of the other components in the mixture. To assess the response to the blend of low abundance components, a second series of trials was conducted for each protein with tricosane omitted from both uninfected and CLas-infected samples, while maintaining the combined concentration of all components at $10 \mu \mathrm{M}$. Each
Table 1 | Common subset of chemical compounds differentially expressed in uninfected and infected citrus plants during different seasons.

\begin{tabular}{|c|c|c|c|c|}
\hline & \multicolumn{2}{|c|}{$\begin{array}{l}\text { Experimental } \\
\text { abundances, au } \times 10^{-4}\end{array}$} & \multicolumn{2}{|c|}{$\begin{array}{l}\text { Molar ratios } \\
\text { in the } \mathrm{mix}\end{array}$} \\
\hline & Uninfected & Infected & Uninfected & Infected \\
\hline Linalool & 8.75 & 12.5 & 11.16 & 11.81 \\
\hline Tridecane & 5.28 & 6.63 & 6.73 & 6.28 \\
\hline 4-OH-4-Me-2-pentanone & 1.21 & 1.06 & 1.54 & 1.00 \\
\hline Hexacosane & 8.34 & 6.53 & 10.64 & 6.18 \\
\hline 1-Tetradecene & 4.03 & 6.34 & 5.14 & 6.01 \\
\hline Tricosane & 92.36 & 64.25 & 117.83 & 60.87 \\
\hline Geranial (Citral) & 1.79 & 10.0 & 2.28 & 9.48 \\
\hline Tetradecanal & 2.52 & 7.61 & 3.22 & 7.21 \\
\hline Phenylacetaldehyde & 6.45 & 8.95 & 8.23 & 8.48 \\
\hline Methyl salicylate & 5.11 & 13.2 & 6.53 & 12.46 \\
\hline Cumacrene* & 0.78 & 3.69 & 1.00 & 3.50 \\
\hline (E)-Beta-ocimene & 8.37 & 5.36 & 10.68 & 5.08 \\
\hline Hexadecanol & 1.21 & 1.06 & 1.54 & 1.00 \\
\hline Geranyl acetone & 25.8 & 46.1 & 32.95 & 43.67 \\
\hline
\end{tabular}

${ }^{*}$ Pure compound was unavailable.

protein was screened with each mixture eight times. A positive control ligand (indole) was used to verify each protein was functional under assay conditions.

The results from the Attenu assays were presented in a form of a fluorescence curve with the wavelength on the abscissa and the fluorescence associated with each wavelength on the ordinate (Figure 2 and Figures A1-A6 in Supplementary Material). In order to verify that statistically significant changes in fluorescence were observed, statistical analysis was performed. Areas under the fluorescence curves were approximated by integration with the trapezoidal rule and the significance of the reduction in fluorescence of each treatment, compared to the protein alone, was assessed with a one-sample $t$-test (Figure 3).

\section{BEHAVIORAL BIOASSAYS}

A two-port divided T-olfactometer (Analytical Research System, Gainesville, FL) was used to evaluate the behavioral response of D. citri to infected ("HLB") and uninfected ("Healthy") odorant mixtures. Chemicals were obtained from the commercial sources as described above. The olfactometer consisted of a vertical $30 \mathrm{~cm}$ long glass tube with $3.5 \mathrm{~cm}$ internal diameter that is bifurcated into two equal halves with a Polytetrafluoroethylene (PTFE) strip forming a T-maze. Each half served as an arm of the olfactometer enabling the $D$. citri to make a choice between two potential odor fields. The chambers containing treatments were attached to inlet and outlet valves for incoming and outgoing air streams, respectively. Purified and humidified air was pushed through these chambers via two pumps connected to an air delivery system at $0.1 \mathrm{~L} / \mathrm{min}$ flow (ARS, Gainesville, FL). A female D. citri was released into the olfactometer and given a choice between two odor sources for $5 \mathrm{~min}$. D. citri were considered non-responsive if they did not make a choice within $5 \mathrm{~min}$. 
Odor sources were randomly assigned to one arm of the olfactometer at the beginning of each bioassay and were reversed every five insects to eliminate positional bias. In addition, prior to odor testing, $D$. citri adult females were exposed to clean air vs. clean air in the olfactometer to verify the absence of positional bias. Response of $D$. citri between the two odor arms in each choice test was assessed with the use of a chi-squared test with an even distribution between odor arms as a null hypothesis.

\section{BEHAVIORAL RESPONSE OF D. CITRI}

The objective of this experiment was to determine whether the "HLB" blend was attractive to $D$. citri when presented against the "Healthy" blend. The odor sources consisted of $24 \mathrm{~cm}$ length volatile collection chambers from Analytical Research Systems (Gainesville, FL) as described by Mann et al. (2011) enclosing a $4 \mathrm{~cm}$ cotton wick. Each cotton wick was impregnated with $100 \mu \mathrm{L}$ of either "HLB" or "Healthy" blend at 0.1 or $0.01 \mu \mathrm{g} / \mu \mathrm{L}$ concentrations. Blends were tested at each concentration against solvent (control) and against each other at both concentrations. This test consisted of 6-8 trials of 15-21 females resulting in a total of 105-165 females tested per treatment combination.

The objective of the second experiment was to determine whether the infected ("HLB") blend was attractive to D. citri when presented against odors from uninfected sweet-orange plants [Citrus sinensis (L.) Osbeck]. Each odor arm consisted of a glass dome (38 cm tall, $15 \mathrm{~cm}$ width, $5 \mathrm{~L}$ ) placed on a PTFE guillotine and attached to volatile collection chambers used in the first behavioral experiment (Figure 1). One arm of the olfactometer received air from a clean and empty glass dome while the collection chamber contained a $4 \mathrm{~cm}$ cotton wick impregnated with $100 \mu \mathrm{L}$ of the "HLB" blend at the $0.1 \mu \mathrm{g} / \mu \mathrm{L}$ dosage (Figure 1). The other arm received odors from a glass dome containing a sweet-orange Valencia plant while the collection chamber simultaneously contained a $4 \mathrm{~cm}$ cotton wick with $100 \mu \mathrm{L}$ of dichloromethane (solvent blank) (Figure 1). The plants were 2years old, between 65 and $90 \mathrm{~cm}$ in height, and pruned 10 days prior to experiments to induce new leaf growth. The PTFE guillotine dome contained between 160 and $200 \mathrm{~cm}^{2}$ of leaf surface and leaf flush were approximately 2.5 and $5 \mathrm{~cm}$ in size within the dome. The leaf surface was calculated according to a standard curve based on the length of each leaf. This test consisted of eight plants used for trials of 20 females, resulting in a total of 160 females tested.

\section{RESULTS}

\section{SELECTION OF CHEMOATTRACTANT COMPOUNDS}

There were 245 statistically discriminating compounds between uninfected and infected trees, based on abundance, identified in the December 2010 samples; 82 discriminating compounds in the March 2011 samples; and 38 discriminating compounds in the September 2011 samples. These compounds overlapped to a large extent with the HLB biomarker compounds reported by Aksenov et al. (2014).

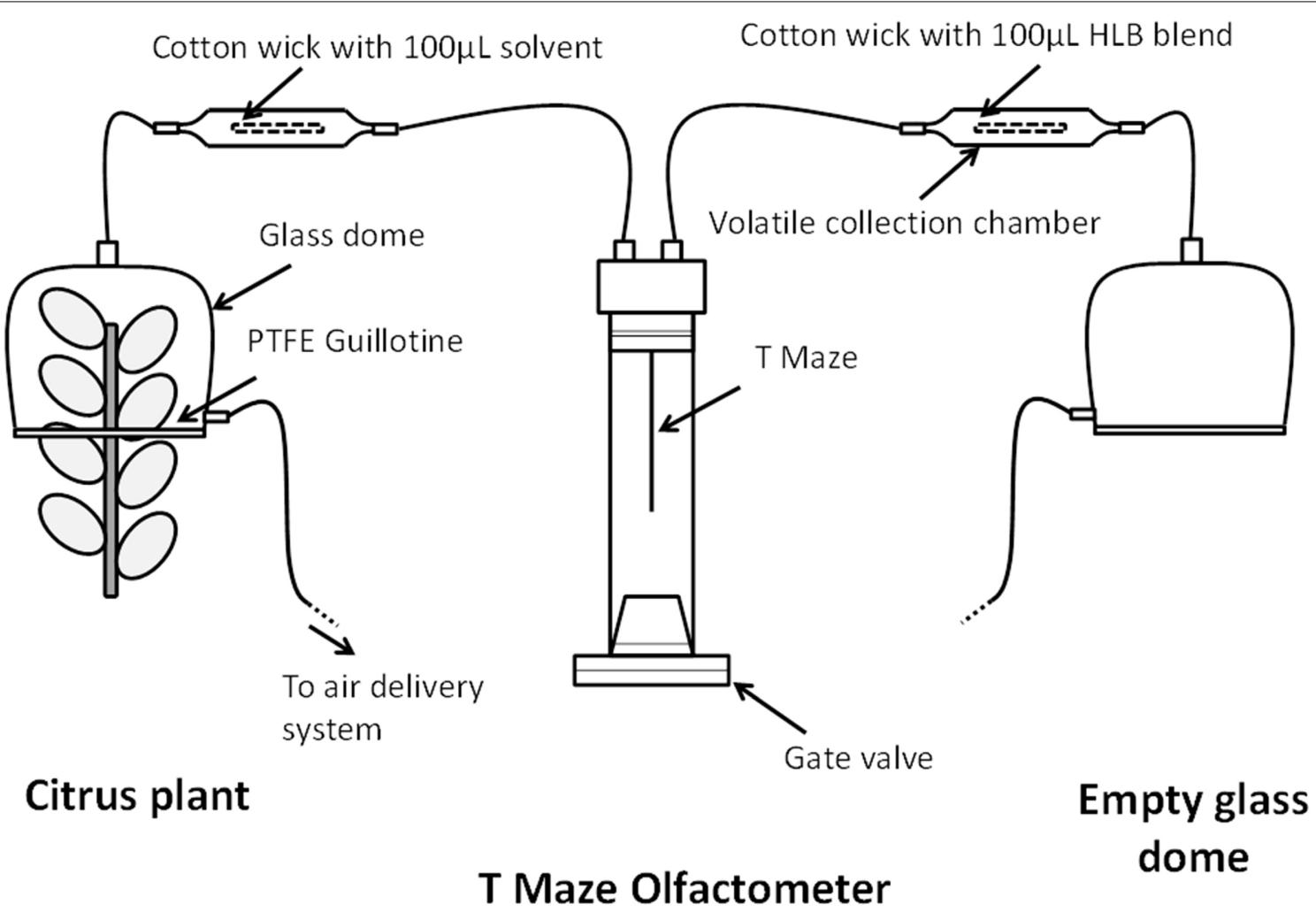

FIGURE 1 | Schematic diagram of the behavioral assay of Diaphirona citri response to the "HLB" (infected) blend vs. citrus plant volatiles. 


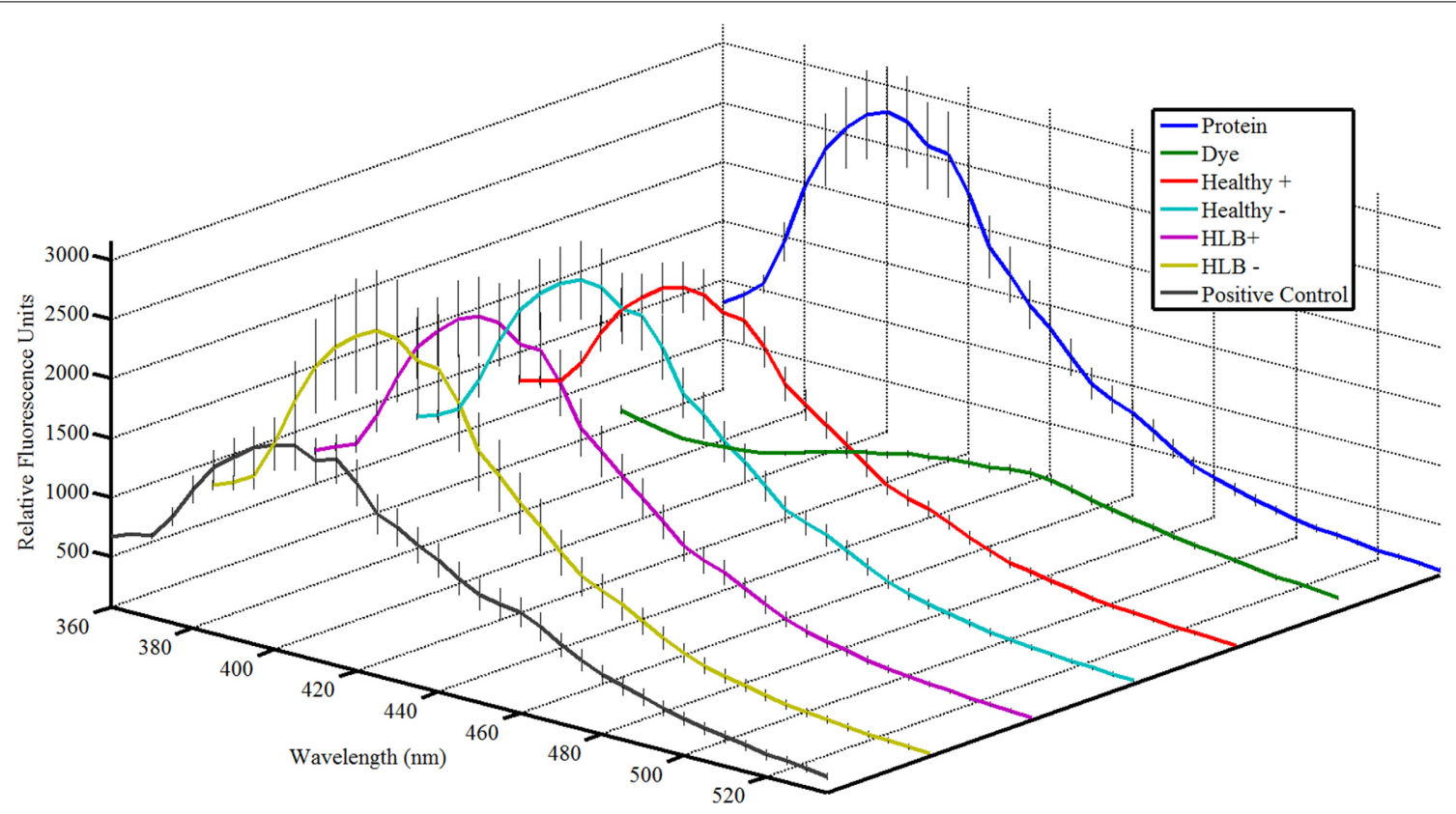

FIGURE 2 | Attenu assay fluorescence plots for mixtures representing semiochemical emissions of $14 \mathrm{HLB}$ biomarkers compounds with the Diaphorina citri chemosensory proteins for DcSAP1 at $10 \mu \mathbf{M}$. Error bars representing one standard deviation are shown. (Protein), protein at corresponding concentration; (Dye), fluorescent dye without protein or ligand; (Healthy +$)$, uninfected mixture with tricosane; (Healthy-), uninfected mixture without tricosane; $(\mathrm{HLB}+)$, HLB mixture with tricosane; (HLB-), HLB mixture without tricosane; (Positive Control), $5 \mu \mathrm{M}$ known ligand (indole). Total concentration of semiochemicals is $10 \mu \mathrm{M}$.
Partial least squares (PLS) regression analysis was applied to quantitatively examine the discrimination power of the selected compounds using a 5 -fold cross-validation strategy (Wold et al., 2001). The systematic classification accuracies between uninfected and infected plants, based on these compounds, were found as follows: $95.0 \%$ ( 53 correctly classified out of 57 for CLasinfected and 62 correctly classified out of 64 for uninfected) for the December 2010 samples; $83.5 \%$ (12 correctly classified out of 20 for uninfected and 54 correctly classified out of 59 for CLas-infected) for the March 2011 samples; 83.3\% (8 correctly classified out of 12 for uninfected and 22 correctly classified out of 24 for HLB-infected) for the September 2011 samples. Thus, these discriminating compounds may be considered as "general purpose" HLB biomarkers, since differences in their abundances were indicative of infection with the CLas pathogen and enabled determining the infection status of citrus trees for all infection stages with relatively high accuracy.

Approximately half of the compounds in Table S1 in the "Supplemental Material" were identified and selected for developing a potential blend attractive to D. citri. The list of these compounds, along with their averaged experimental abundances, is given in Table 1. For the purpose of discriminating between uninfected and infected trees, based on the abundances of common compounds, detailed lists of biomarkers are given in Table S1 in the "Supplemental Material". The classification accuracy remains nearly unchanged. For example, 93.4\% (54/57 for HLB-infected and 59/64 for uninfected) for the December 2010 samples and 83.5\% (10/20 for uninfected and 56/59 for CLas-infected) for the March 2011 samples. These results indicate that this "universal" biomarker subset, although very limited compared to the total number of volatiles produced by trees, is still sufficient to discern CLas infection with high accuracy.

\section{SCREENING D. CITRI CHEMOSENSORY PROTEINS WITH COMPOUND MIXTURES USING THE ATTENU ASSAY SYSTEM}

The Attenu assays indicated that none of the compounds bound to any of the $D$. citri chemosensory proteins when tested individually (data not shown). However, the equimolar mixture of all compounds did show moderate binding to DcOBP1 (Figure A1 in Supplementary Material). Mixtures were created with ratios of compounds as listed in Table 1 (total concentration of all compounds $10 \mu \mathrm{M}$ ) with the intention to represent typical semiochemical emissions from uninfected or infected trees under assay conditions. The summary of assay results is shown in Figure 3 and all the experimental data are presented in Figures A2-A6 in the "Supplemental Material." An example of an assay result for the DcSAP1 protein is shown in Figure 2. The results indicate interaction between the "infected" mixture without tricosane and DcOBP1 $\left[t_{(7)}=3.37, P=0.012\right.$; Figure 3A, Figure A2). DcSAP1 bound strongest the uninfected ("Healthy") plant mixture both in the presence $\left[t_{(7)}=8.38, P<0.001\right]$ and absence of tricosane $\left[t_{(7)}=3.074, P=0.018\right]$, and to a lesser extent the "infected" mixture with tricosane $\left[t_{(7)}=2.31, P=\right.$ 0.054; Figure 3B, Figures A2, A3). DcSAP2 showed interaction with the uninfected ("Healthy") mixture, both in the presence $\left[t_{(7)}=2.80, P=0.027\right]$ and absence of tricosane $\left[t_{(7)}=2.62\right.$, $P=0.034$; Figure 3C, Figure A4]. DcSAP3 bound the uninfected 

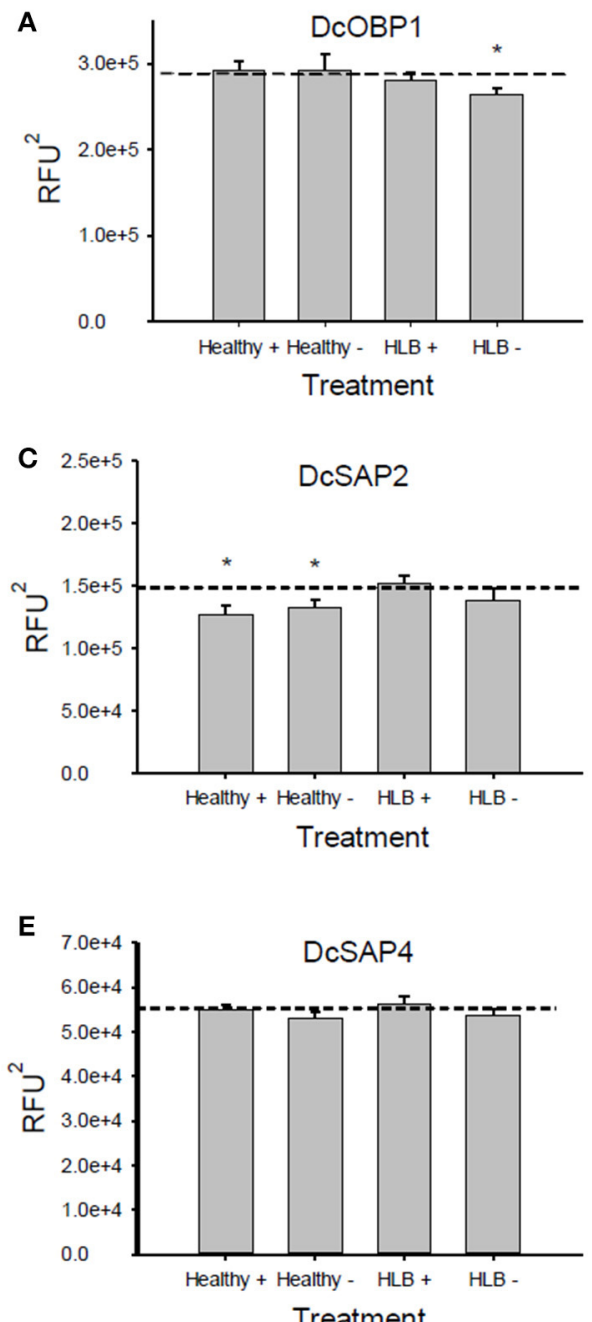

FIGURE 3 | Average values ( \pm SE) of the areas under the fluorescence curves for the Attenu assays. A reduction of the fluorescence compared to the average fluorescence of the protein alone (dotted lines) indicates an interaction between the protein and the mixture tested. (A) DcOBP1, (B) DcSAP1, (C) DcSAP2, (D) DcSAP3,

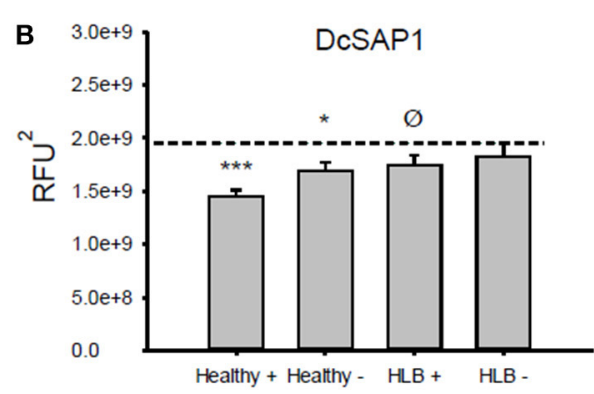

Treatment

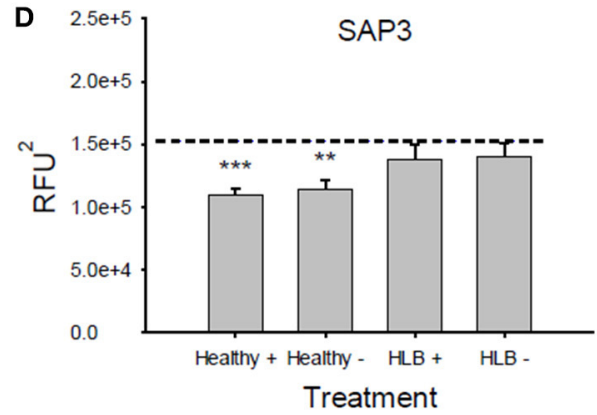

(E) DcSAP4. (Healthy+), uninfected mixture with tricosane. (Healthy-), uninfected mixture without tricosane; (HLB+), HLB mixture with tricosane; (HLB-), HLB mixture without tricosane. Total concentration of semiochemicals is $10 \mu \mathrm{M}$. ${ }^{* * *} P<0.001,{ }^{* *} P<0.01,{ }^{*} P<0.05,{ }^{\varnothing} P<0.1$. RFU2: Relative fluorescence units ${ }^{2}$. Note differences in $y$-axes scales.
("Healthy") mixture both in the presence $\left[t_{(7)}=8.92, P<0.001\right]$ and absence of tricosane $\left[t_{(7)}=5.15, P=0.001\right.$; Figure $3 \mathrm{D}$, Figure A5]. DcSAP4 did not interact with any of the mixtures tested (Figure 3E, Figure A6).

\section{BEHAVIORAL BIOASSAYS}

We tested the behavioral response of $D$. citri to these artificial chemical blends with a vertical T-maze olfactometer (Mann et al., 2011). Single D. citri females were introduced into the olfactometer and were given the choice between two odor arms. Female D. citri were not attracted to the uninfected ("Healthy") blend as compared to the solvent blank at both dosages tested $(0.01 \mu \mathrm{g} / \mu \mathrm{L}: \chi=1.15, n=105$, d.f. $=1, p=0.283 ; 0.1 \mu \mathrm{g} / \mu \mathrm{L}$ : $\chi=0.82, n=78$, d.f. $=1, p=0.365)$. Female $D$. citri were not attracted to the infected ("HLB") blend at $0.01 \mu \mathrm{g} / \mu \mathrm{L}(\chi=0.07$, $n=129$, d.f. $=1, p=0.792)$, but were attracted to this blend at the $0.1 \mu \mathrm{g} / \mu \mathrm{L}$ dosage $(\chi=5.04, n=134$, d.f. $=1, p=0.025)$ as compared with the solvent blank.

Female D. citri were consistently attracted to the infected ("HLB") blend as compared with the uninfected ("Healthy") blend at both the $0.01 \mu \mathrm{g} / \mu \mathrm{L}(\chi=8.31, n=131$, d.f. $=1$, $p=0.004)$ and the $0.1 \mu \mathrm{g} / \mu \mathrm{L}(\chi=5.14, n=103$, d.f. $=1$, $p=0.023)$ dosages. Similarly, $D$. citri were significantly more attracted to the synthetic infected ("HLB") blend than the natural odor from uninfected sweet orange citrus trees $(X=4.35$, $n=92$, d.f. $=1, p=0.037)$. Only the synthetic "HLB" blend attracted $D$. citri as compared with a blank solvent at the concentrations tested here. Also, D. citri were consistently attracted to the synthetic, multi-component "HLB" blend as compared with odors consistent with uninfected plants. This occurred when 
response of $D$. citri was compared between the "HLB" synthetic blend versus a synthetic blend mimicking uninfected trees (Figure 4). Also, D. citri were more attracted to the synthetic "HLB" blend than to odors from uninfected citrus trees serving as the controls (Figure 4).

\section{DISCUSSION}

Herein we attempted to develop an attractant for $D$. citri by identifying and mimicking the chemical cues produced by HLBinfected citrus trees. The chemical signature of sweet orange citrus trees infected with the CLas pathogen that causes HLB has been previously elucidated (Aksenov et al., 2014). The VOC profiling of citrus plants was conducted throughout an entire year in three independent studies across different growing seasons. It was demonstrated that overall VOC distributions changed significantly by season. Both uninfected and CLas-infected trees were characterized by complex VOC"landscapes" (Aksenov et al., 2014). The compounds with different abundances among uninfected and HLB-infected plants (potential HLB biomarkers) were also found to be season-specific in release. Seasonal changes in plant metabolism coupled with fluctuating titer of CLas (Manjunath et al., 2008) and differences in the pathogen's life cycle (e.g., differential expression of CLas genes, Yan et al., 2013) may contribute to annual changes in VOC profiles. It would be challenging and likely impractical to create such complex and dynamic mixtures of hundreds of compounds for use as insect lures. However, it is likely that only a subset of compounds emitted by citrus plants is necessary to affect $D$. citri behavior.

We focused on a short list of compounds that we selected as "universal" HLB biomarkers (Table 1, Table S1 in the "Supplemental Material") for assaying behavior of D. citri. Developing a lure blend with only a subset of compounds is practical, but it is a limited representation of the entire citrus VOC profile, especially since almost a half of compounds in Table S1 in the "Supplemental Material" were not identified. However, the identified compounds in this subset were previously implicated as D. citri attractants, most notably, methyl salicylate (MeSA) (Mann et al., 2012). Some of the identified compounds in Table 1 (e.g., linalool, $\beta$-ocimene) were identified as semiochemicals attractive to $D$. citri as they are produced by young leaf flush of rutaceous plants. Since young leaves are the primary feeding and egg laying resource for D. citri (Patti and Setamou, 2010), it is presumed that $D$. citri preferentially select young leaves using olfactory and visual cues. Thus, alteration of abundances of these compounds due to CLas infection may be responsible for even greater attraction of $D$. citri to critical resources on infected plants (Eigenbrode et al., 2002; McLeod et al., 2005; Mauck et al., 2010; Davis et al., 2012; Shapiro et al., 2012).

In order to investigate whether the selected compounds may be active at the peripheral level, we tested each of the 14 compounds given in Table 1 with the Attenu assay system (Pelosi et al., 2006) with five chemosensory proteins from D. citri, as described above. The assay revealed that no individual compound interacted with any of the tested proteins. One interpretation is that the available chemosensory proteins were not capable of binding these particular individual compounds in vivo or the response was not elicited by a single compound and, perhaps, binding of multiple compounds was required. To explore the latter possibility, we then tested an equimolar mixture of all 14 compounds to all five available odorant binding proteins. In the case of DcOBP1, we observed a moderate interaction between the proteins and the compound mixture (Figure A1 in Supplementary Material). Consequently, we further tested the mixtures intended to represent typical semiochemical emissions from uninfected and infected trees. We found that the mixtures of biomarkers with concentrations approximately corresponding to their gas-phase abundances in the field studies produced a response from more than one chemosensory protein (Figure 3 and Figures A2-A6 in Supplementary Material). Our assay screening results indicated that a mixture of semiochemicals was necessary for the selected chemical biomarkers to elicit an odorant binding response in D. citri. Typically numerous ligands, both natural and/or synthetic, may produce response for any given OBP. There are several examples of crystal structures of OBP proteins, and dimerization of OBPs has also been noted (Briand et al., 2001; Wogulis et al., 2006; Leite et al., 2009; Tsitsanou et al., 2013). These dimers may affect individual component conformations, as well as, lead to the formation of a "third" binding pocket. The above factors may have contributed to the observed effect.

The Attenu technique allowed for rapid screening of functionality of a complex blend of semiochemicals based on volatile changes that occurred because of pathogen infection. The elicited response appears to be more pronounced for the uninfected ("Healthy") blend as compared to the infected blend.

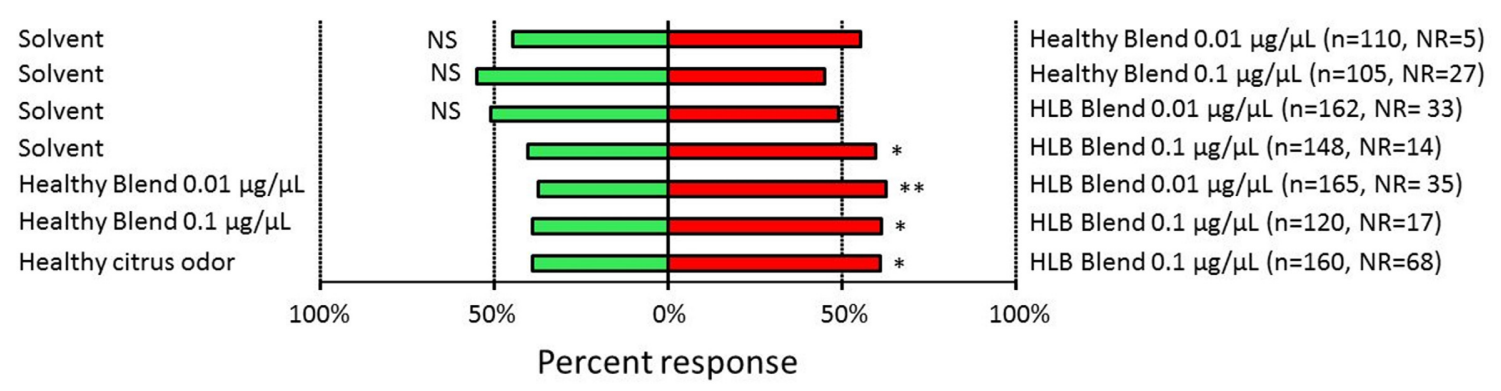

FIGURE 4 | Responses of Diaphorina citri when presented with volatiles emanating from "Healthy" (uninfected), "HLB" (infected) synthetic blends or from uninfected citrus plant volatiles. NR, Non responder. Stars indicate significant difference between treatments. ${ }^{*} P<0.05$, ${ }^{* *} P<0.01$. 
However, the results of this assay could only be interpreted as to whether the insect's odorant binding proteins interacted with the semiochemical blend. The Attenu assay is designed to be a fast screening tool. A positive result indicates that the substrate is relevant; however, this does not necessarily translates into systemic perception of the chemical by the insect, nor does it mean that the insect's behavior would be affected. On the other hand, a negative result could indicate that the available chemosensory proteins are not responsible for recognition of the particular compounds tested in vivo. However, due to the nature of the assay system, a negative result will not disprove that the target organism can detect a tested compound, nor will it reveal what, if any, behavioral effects a compound will have on that organism. Further behavioral assays are necessary to determine the effect of semiochemicals on the insect's behavior. Typically, only a limited number of substrates are found to elicit response in Attenu assays. Thus, it is highly unlikely that the exhibited interaction of compound blends with more than one protein is accidental. The fact that the Attenu assay results corroborate the results of the behavioral tests suggests that the observed activity of the attractant described here for D. citri is not circumstantial. Preferential attraction of $D$. citri to the infected blend as compared with the uninfected blend determined in behavioral assays suggests the importance of identifying a precise abundance ratio of semiochemicals for attraction of $D$. citri to pathogen-infected plants.

MeSA alone is an attractant for D. citri (Mann et al., 2012). However, under field conditions, D. citri must discriminate among bouquets of volatiles and complex mixtures are likely more important for host location than individual compounds (Webster et al., 2010). Therefore, we hypothesize that a mixture of compounds that identifies HLB-infected plants is likely more important for location of infected hosts by $D$. citri than individual chemicals. The current research indicates that uninfected trees released less MeSA as compared with infected counterparts. This suggests that MeSA induction is not restricted to HLB infection and likely not the sole indicator of infected plants for D. citri. For example, herbivore damage is known to cause release of MeSA in citrus (Mann et al., 2012; Martini et al., 2014) and other causes of damage or stress may also induce release of this volatile. Consequently, we suggest that a complex blend of chemicals rather than MeSA alone, may be required for $D$. citri to select an infected plant over an uninfected one in a natural field setting. However, direct field testing with formulated lures will be needed to test this hypothesis.

The blends identified from both uninfected and infected plants were comprised of the same compounds with only relatively small differences in abundances of constituents $(\sim 1.5-2$-fold, $\sim 4$-fold for geranial). Yet, these small differences caused specific attraction of $D$. citri to the blend characteristic of infected plants. Identification of the specific abundances of components in the blend mixture may help avoid a trial and error approach for lure development. The idea of implementing semiochemicals for insect management is common, with significant efforts focused on insect monitoring and pheromone-based mating disruption (Witzgall et al., 2010a). Indirect control of pest populations through the use of herbivore-induced plant volatiles to attract carnivorous arthropods has also shown promise (Kaplan, 2012). The idea of "attract and reward" for conservation biological control has also been investigated (Gordon et al., 2013). We present an approach for semiochemical identification that considers analytical chemistry, protein binding assays at the peripheral nervous system level, and behavioral assays. Although we have shown feasibility of the approach only for citrus and D. citri, a similar approach may be useful for developing lures for vectors of other phytopathogens. The currently identified attractive blend may have use for monitoring or attract-and-kill of $D$. citri. The possible practical application of the blend identified here will need to be elucidated in subsequent field-based investigations.

\section{ACKNOWLEDGMENTS}

The authors are grateful to Inscent, Inc. for conducting the fluorescence-based assays. This study was supported by the California Citrus Research Board (CRB) [5100-143 Cristina E. Davis; 5500-206 Cristina E. Davis, Lukasz L. Stelinski, Alexander A. Aksenov], the Industry-University Cooperative Research Program (UC Discovery) [Cristina E. Davis], and the Florida Citrus Production Advisory Council (FCPRAC) [Cristina E. Davis] as well as by the Citrus Research and Development Foundation (CRDF) [Lukasz L. Stelinski, Xavier Martini]. We thank Angelique Hoyte, Laurie Martini and Wendy Meyer for their help in the behavioral assays, and Sierra Spitulski for assistance with figures 2 and A2-A6 preparation.

\section{SUPPLEMENTARY MATERIAL}

The Supplementary Material for this article can be found online at: http://www.frontiersin.org/journal/10.3389/fevo.2014.00078/ abstract

\section{REFERENCES}

Aksenov, A. A., Pasamontes, A., Peirano, D. J., Zhao, W., Dandekar, A. M., Fiehn, O., et al. (2014). Detection of huanglongbing disease using differential mobility spectrometry. Anal. Chem. 86, 2481-2488. doi: 10.1021/ac403469y

Biessmann, H., Andronopoulou, E., Biessmann, M. R., Douris, V., Dimitratos, S. D., Eliopoulos, E., et al. (2010). The Anopheles gambiae Odorant Binding Protein 1 (AgamOBP1) mediates indole recognition in the antennae of female mosquitoes. PLoS ONE 5:e9471. doi: 10.1371/journal.pone.0009471

Briand, L., Nespoulous, C., Huet, J. C., Takahashi, M., and Pernollet, J. C. (2001). Ligand binding and physico-chemical properties of ASP2, a recombinant odorant-binding protein from honeybee (Apis mellifera L.). Eu. J. Biochem. 268, 752-760. doi: 10.1046/j.1432-1327.2001.01927.x

Callaway, E. (2008). Bioterror: The green menace. Nature 452, 148-150. doi: $10.1038 / 452148 \mathrm{a}$

Davis, T. S., Horton, D. R., Munyaneza, J. E., and Landolt, P. J. (2012). Experimental infection of plants with an herbivore-associated bacterial endosymbiont influences herbivore host selection behavior. PLOS ONE 7:e49330. doi: 10.1371/ journal.pone.0049330

Deng, Y. H., Yan, H., Gu, J. B., Xu, J. B., Wu, K., Tu, Z. J., et al. (2013). Molecular and functional characterization of odorant-binding protein genes in an invasive vector mosquito, Aedes albopictus. PLoS ONE 8:e68836. doi: 10.1371/journal.pone.0068836

Eigenbrode, S. D., Ding, H. J., Shiel, P., and Berger, P. H. (2002). Volatiles from potato plants infected with potato leafroll virus attract and arrest the virus vector, Myzus persicae (Homoptera: Aphididae). Proc. R. Soc. B. 269, 455-460. doi: 10.1098/rspb.2001.1909

Gordon, G. U. S. O., Wratten, S. D., Jonsson, M., Simpson, M., and Hale, R. (2013). "Attract and reward": combining a herbivore-induced plant volatile with floral resource supplementation - Multi-trophic level effects. Biol. Control 64, 106-115. doi: 10.1016/j.biocontrol.2012.10.003 
Grafton-Cardwell, E. E., Stelinski, L. L., and Stansly, P. A. (2013). Biology and management of Asian citrus psyllid, vector of the huanglongbing pathogens. Annu. Rev. Entomol. 58, 413-432. doi: 10.1146/annurev-ento-120811-153542

Kaplan, I. (2012). Attracting carnivorous arthropods with plant volatiles: the future of biocontrol or playing with fire? Biol. Control 60, 77-89. doi: 10.1016/j.biocontrol.2011.10.017

Kos, M., Houshyani, B., Overeem, A. J., Bouwmeester, H. J., Weldegergis, B. T., Van Loon, J. J. A., et al. (2013). Genetic engineering of plant volatile terpenoids: effects on a herbivore, a predator and a parasitoid. Pest Manag. Sci. 69, 302-311. doi: $10.1002 / \mathrm{ps} .3391$

Kost, C., and Heil, M. (2006). Herbivore-induced plant volatiles induce an indirect defence in neighbouring plants. J. Ecol. 94, 619-628. doi: 10.1111/j.13652745.2006.01120.x

Leite, N. R., Krogh, R., Xu, W., Ishida, Y., Iulek, J., Leal, W. S., et al. (2009). Structure of an odorant-binding protein from the mosquito Aedes aegypti suggests a binding pocket covered by a pH-sensitive "Lid". PLoS ONE 4:e8006. doi: 10.1371/ journal.pone.0008006

Mann, R. S., Ali, J. G., Hermann, S. L., Tiwari, S., Pelz-Stelinski, K. S., Alborn, H. T., et al. (2012). Induced release of a plant-defense volatile "deceptively" attracts insect vectors to plants infected with a bacterial pathogen. PLoS Pathog. 8:e1002610. doi: 10.1371/journal.ppat.1002610

Mann, R. S., Rouseff, R. L., Smoot, J. M., Castle, W. S., and Stelinski, L. L. (2011). Sulfur volatiles from Allium spp. affect Asian citrus psyllid, Diaphorina citri Kuwayama (Hemiptera: Psyllidae), response to citrus volatiles. Bull. Entomol. Res. 101, 89-97. doi: 10.1017/S0007485310000222

Matsuo, T., Sugaya, S., Yasukawa, J., Aigaki, T., and Fuyama, Y. (2007). Odorantbinding proteins OBP57d and OBP57e affect taste perception and host-plant preference in Drosophila sechellia. PLoS Biol. 5:e118. doi: 10.1371/journal.pbio. 0050118

Manjunath, K. L., Halbert, S. E., Ramadugu, C., Webb, S., and Lee, R. F. (2008). Detection of'Candidatus Liberibacter asiaticus' in Diaphorina citri and its importance in the management of Citrus huanglongbing in Florida. Phytopathology 98, 387-396. doi: 10.1094/PHYTO-98-4-0387

Martini, X., Kuhns, E. H., Hoyte, A., and Stelinski, L. L. (2014). Plant volatiles and density-dependent conspecific female odors are used by Asian citrus psyllid to evaluate host suitability on a spatial scale. Arthropod-Plant Inte. 8, 453-460. doi: 10.1007/s11829-014-9326-z

Mauck, K. E., De Moraes, C. M., and Mescher, M. C. (2010). Deceptive chemical signals induced by a plant virus attract insect vectors to inferior hosts. Proc. Nat. Aca. Sci. U.S.A. 107, 3600-3605. doi: 10.1073/pnas.0907191107

McLeod, G., Gries, R., von Rueuss, S. H., Rahe, J. E., McIntosh, R., Konig, W. A., et al. (2005). The pathogen causing Dutch elm disease makes host trees attract insect vectors. Proc. R. Soc. B. 272, 2499-2503. doi: 10.1098/rspb.2005.3202

Patti, J. M., and Setamou, M. (2010). Responses of the Asian citrus psyllid to volatiles emitted by the flushing shoots of its rutaceous host plants. Environ. Entomol. 39, 618-624. doi: 10.1603/EN09216

Pelletier, J., Guidolin, A., Syed, Z., Cornel, A. J., and Leal, W. S. (2010). Knockdown of a mosquito odorant-binding protein involved in the sensitive detection of oviposition attractants. J. Chem. Ecol. 36, 245-248. doi: 10.1007/s10886-0109762-x

Pelosi, P., Zhou, J. J., Ban, L. P., and Calvello, M. (2006). Soluble proteins in insect chemical communication. Cell. Mol. Life Sci. 63, 1658-1676. doi: 10.1007/s00018-005-5607-0

Pelz-Stelinski, K. S., Brlansky, R. H., Ebert, T. A., and Rogers, M. E. (2010). Transmission parameters for 'Candidatus Liberibacter asiaticus' by Asian citrus psyllid (Hemiptera: Psyllidae). J. Econ. Entomol. 103, 1531-1541. doi: 10.1603/ EC10123

Pierik, R., Ballare, C. L., and Dicke, M. (2014). Ecology of plant volatiles: taking a plant community perspective. Plant Cell Env. 37, 1845-1853. doi: 10.1111/pce. 12330
Rodriguez-Saona, C., Kaplan, I., Braasch, J., Chinnasamy, D., and Williams, L. (2011). Field responses of predaceous arthropods to methyl salicylate: a metaanalysis and case study in cranberries. Biol. Control 59, 294-303. doi: 10.1016/j. biocontrol.2011.06.017

Shapiro, L., De Moraes, C. M., Stephenson, A. G., and Mescher, M. C. (2012). Pathogen effects on vegetative and floral odours mediate vector attraction and host exposure in a complex pathosystem. Ecol. Lett. 15, 1430-1438. doi: 10.1111/ ele. 12001

Skogerson, K., Wohlgemuth, G., Barupal, D. K., and Fiehn, O. (2011). The volatile compound BinBase mass spectral database. BMC Bioinform. 12:321. doi: 10.1186/1471-2105-12-321

Swarup, S., Williams, T. I., and Anholt, R. R. H. (2011). Functional dissection of Odorant binding protein genes in Drosophila melanogaster. Genes Brain Behav. 10, 648-657. doi: 10.1111/j.1601-183X.2011.00704.x

Tsitsanou, K. E., Drakou, C. E., Thireou, T., Gruber, A. V., Kythreoti, G., Azem, A., et al. (2013). Crystal and solution studies of the "Plus-C" odorant-binding protein 48 from Anopheles gambiae control of binding specificity through threedimensional domain swapping. J. Biol. Chem. 288, 33427-33438. doi: 10.1074/ jbc.M113.505289

Webster, B., Bruce, T., Pickett, J., and Hardie, J. (2010). Volatiles functioning as host cues in a blend become nonhost cues when presented alone to the black bean aphid. Anim. Behav. 79, 451-457. doi: 10.1016/j.anbehav.2009.11.028

Witzgall, P., Kirsch, P., and Cork, A. (2010a). Sex pheromones and their impact on pest management. J. Chem. Ecol. 36, 80-100. doi: 10.1007/s10886-009-9737-y

Witzgall, P., Trematerra, P., Liblikas, I., Bengtsson, M., and Unelius, C. R. (2010b). Pheromone communication channels in tortricid moths: lower specificity of alcohol vs. acetate geometric isomer blends. Bull. Entomol. Res. 100, 225-230. doi: 10.1017/S0007485309990186

Wogulis, M., Morgan, T., Ishida, Y., Leal, W. S., and Wilson, D. K. (2006). The crystal structure of an odorant binding protein from Anopheles gambiae: evidence for a common ligand release mechanism. Biochem. Bioph. Res. Co. 339, 157-164. doi: 10.1016/j.bbrc.2005.10.191x

Wold, S., Sjöström, M., and Eriksson, L. (2001). PLS-regression: a basic tool of chemometrics. Chemometr. Intell. Lab. 58, 109-130.

Yan, Q., Sreedharan, A., Wei, S., Wang, J., Pelz-Stelinski, K., Folimonova, S., et al. (2013). Global gene expression changes in Candidatus Liberibacter asiaticus during the transmission in distinct hosts between plant and insect. Mol. Plant Pathol. 14, 391-404. doi: 10.1111/mpp.12015

Zhou, J. J., Zhang, G. A., Huang, W. S., Birkett, M. A., Field, L. M., Pickett, J. A., et al. (2004). Revisiting the odorant-binding protein LUSH of Drosophila melanogaster: evidence for odour recognition and discrimination. FEBS Lett. 558, 23-26. doi: 10.1016/S0014-5793(03)01521-7

Conflict of Interest Statement: The authors declare that the research was conducted in the absence of any commercial or financial relationships that could be construed as a potential conflict of interest.

Received: 06 June 2014; accepted: 19 November 2014; published online: 09 December 2014.

Citation: Aksenov AA, Martini X, Zhao W, Stelinski LL and Davis CE (2014) Synthetic blends of volatile, phytopathogen-induced odorants can be used to manipulate vector behavior. Front. Ecol. Evol. 2:78. doi: 10.3389/fevo.2014.00078

This article was submitted to Chemical Ecology, a section of the journal Frontiers in Ecology and Evolution.

Copyright (C) 2014 Aksenov, Martini, Zhao, Stelinski and Davis. This is an openaccess article distributed under the terms of the Creative Commons Attribution License (CC BY). The use, distribution or reproduction in other forums is permitted, provided the original author(s) or licensor are credited and that the original publication in this journal is cited, in accordance with accepted academic practice. No use, distribution or reproduction is permitted which does not comply with these terms. 